\title{
Computer Simulation of Bacterial Cultures
}

\author{
WILLIAM D. YOUNG, JR. \\ Department of Biology, Fairleigh Dickinson University, Madison, New Jersey 07940
}

\begin{abstract}
A method is described for the simulation of bacterial biochemical taxonomic data with randomly inserted variations from typical reactions. This method is applied to data from a group of Enterobacteriaceae. The simulated data thus generated show good agreement with expected results. The procedure will be useful in evaluating identification algorithms and in analysis of patterns of variation among bacteria.
\end{abstract}

In biological taxonomy, it is sometimes helpful to analyze artificial organisms possessing the types of variation which are under study. In zoology, the "Caminalcules," a group of hypothetical animals, are used for this purpose (1, $14,15)$. Numerical methods are a valuable aid in the study and definition of bacterial taxa, particularly those taxa which cannot be identified by any single characteristic, but rather are defined by a number of shared characteristics (i.e., polythetic taxa) $(16,17)$. As an adjunct to the development and evaluation of a program for computer-assisted identification of bacterial species, it was considered desirable to generate some hypothetical cultures. Each hypothetical culture simulates an individual bacterial isolate, such as might be obtained from a pathological or environmental specimen. All of the hypothetical cultures consist of coded results of a series of standard biochemical tests that might have been performed on living cultures in order to identify them.

The advantages of simulation are as follows. (i) A larger number of cultures are generated than could readily be assembled from living material. (ii) The time required to produce these hypothetical cultures is vastly less than the time it would take to perform the corresponding biochemical tests. (iii) Rare species can be simulated in numbers equal to those of commonly found species. Thus, an identification procedure can be evaluated as thoroughly for the rare species as for the common ones. (iv) In assembling a data base for this study, figures have been compiled from the literature which represent a very large sample of individual isolates. In the case of rare species, a sizable fraction of all known occurrences can be included. Such large and broadly based samples provide for a more representative measurement of species variability than smaller samples derived from a limited geographical area. (v) The identification program (manuscript in preparation) uses the same data base as those employed in producing the hypothetical cultures. Thus, when the hypothetical cultures are presented to the identification program, optimal conditions exist for their correct identification. This makes it easier to check the identification program and improve its accuracy in subsequent modifications.

(This work was modified from a portion of a thesis submitted to Fairleigh Dickinson University in partial fulfillment of the requirements for the degree of Master of Science.)

\section{MATERIALS AND METHODS}

In the present study, a data base was compiled from the literature (3-7, 11-13) on 23 taxonomic units (genera and species) of Enterobacteriaceae (Table 1, footnote $a$ ). The expected percent positive for 28 two-state (positive or negative) biochemical tests (Table 1, footnote $b$ ) was tabulated for each taxonomic unit. These data (Table 1) represent variation among isolates within the taxonomic units, including any analytical variation which occurred. (For example, $98.6 \%$ of all Escherichia coli strains reported in these data were indole positive.)

The hypothetical cultures were constructed by a simulation program using a random-number generator to select positive and negative test results in proportion to their incidence as defined by the data base. The generator was applied separately to each cell of the data matrix (Table 1), so that each simulated culture test result was independent. The simulation program operated as follows. Each percent positive value was multiplied by a factor of 10 , to convert it to an integer with a range from 0 to 1,000 (i.e., $100.0 \%=1,000$ ). A uniform random-number generator (subroutine RANDU, specific for IBM System $/ 360$ ) was used to generate an integer from 0 to 1,000 . The generated random number and the percent positive integer were then compared. If the random number was smaller, the result of this simulated biochemical test was recorded as positive. If the random number was equal or larger, a negative result was recorded. This procedure was performed for all 
TABLE 1. Data base. Expected percent positive reactions of 23 taxa $^{a}$ in 28 biochemical tests ${ }^{b} \mathrm{c}$

\begin{tabular}{|c|c|c|c|c|c|c|c|c|}
\hline \multirow{2}{*}{$\begin{array}{l}\text { Biochemical } \\
\text { tests }\end{array}$} & \multicolumn{8}{|c|}{ Bacterial taxa ${ }^{\alpha}$} \\
\hline & ESCH & SHIG & EDWR & CSUI & TYPH & ENTR & ARIZ & CITR \\
\hline IND & 98.6 & 39.8 & 99.1 & 0.0 & 0.0 & 1.3 & 2.0 & 6.7 \\
\hline M-R & 99.9 & 100.0 & 100.0 & 100.0 & 100.0 & 100.0 & 100.0 & 99.5 \\
\hline V-P & 0.0 & 0.0 & 0.0 & 0.0 & 0.0 & 0.0 & 0.0 & 0.0 \\
\hline CIT & 0.2 & 0.0 & 0.0 & 60.0 & 0.0 & 99.3 & 98.7 & 90.4 \\
\hline $\mathrm{H}_{2} \mathrm{~S}$ & 0.0 & 0.0 & 99.7 & 60.0 & 94.3 & 98.0 & 98.7 & 81.6 \\
\hline URE & 0.0 & 0.0 & 0.0 & 0.0 & 0.0 & 0.0 & 0.0 & 69.4 \\
\hline $\mathrm{KCN}$ & 2.4 & 0.0 & 0.0 & 0.0 & 0.0 & 0.6 & 8.7 & 96.2 \\
\hline MOT & 69.1 & 0.0 & 98.2 & 100.0 & 100.0 & 100.0 & 100.0 & 95.7 \\
\hline GEL & 0.0 & 0.0 & 0.0 & 0.0 & 0.0 & 0.0 & 4.0 & 0.0 \\
\hline LYS & 88.7 & 0.0 & 100.0 & 95.0 & 100.0 & 99.7 & 100.0 & 0.0 \\
\hline ARG & 17.6 & 9.5 & 0.0 & 0.0 & 81.3 & 69.5 & 12.7 & 43.6 \\
\hline ORN & 64.2 & 20.0 & 100.0 & 100.0 & 0.0 & 99.7 & 100.0 & 17.2 \\
\hline P-D & 0.0 & 0.0 & 0.0 & 0.0 & 0.0 & 0.0 & 0.0 & 0.0 \\
\hline MAL & 0.0 & 0.0 & 0.0 & 0.0 & 0.0 & 0.6 & 92.6 & 21.8 \\
\hline GAS & 91.1 & 2.1 & 99.4 & 100.0 & 0.0 & 97.7 & 99.3 & 90.9 \\
\hline LAC & 90.8 & 0.3 & 0.0 & 0.0 & 0.0 & 0.9 & 61.3 & 39.4 \\
\hline SUC & 48.9 & 0.9 & 0.3 & 0.0 & 0.0 & 0.6 & 4.7 & 15.3 \\
\hline MAN & 96.8 & 80.5 & 0.0 & 100.0 & 100.0 & 100.0 & 100.0 & 99.8 \\
\hline DUL & 49.5 & 5.4 & 0.0 & 5.0 & 0.0 & 98.3 & 0.0 & 59.4 \\
\hline SAL & 40.0 & 0.0 & 0.0 & 0.0 & 0.0 & 0.0 & 4.7 & 4.1 \\
\hline $\mathrm{ADO}$ & 5.6 & 0.0 & 0.0 & 0.0 & 0.0 & 0.0 & 0.0 & 0.0 \\
\hline INO & 1.1 & 0.0 & 0.0 & 0.0 & 0.0 & 42.8 & 0.0 & 3.3 \\
\hline SOR & 93.4 & 29.1 & 0.3 & 85.0 & 100.0 & 99.7 & 97.0 & 98.0 \\
\hline ARA & 99.4 & 67.8 & 9.3 & 0.0 & 0.0 & 99.3 & 98.0 & 100.0 \\
\hline RAF & 50.9 & 20.7 & 0.0 & 0.0 & 0.0 & 3.5 & 5.0 & 14.3 \\
\hline RHM & 82.3 & 16.6 & 0.0 & 100.0 & 0.0 & 95.0 & 93.0 & 99.4 \\
\hline ONP & 95.9 & 13.5 & 0.0 & 0.0 & 0.0 & 2.1 & 100.0 & 74.4 \\
\hline ESC & 30.9 & 0.0 & 0.0 & 0.0 & 0.0 & 0.0 & 0.0 & 0.9 \\
\hline \multirow{2}{*}{$\begin{array}{l}\text { Biochemical } \\
\text { tests }\end{array}$} & \multicolumn{8}{|c|}{ Bacterial taxa } \\
\hline & KPNU & KOZE & KRHI & ECLO & EAER & EHAF & ELIQ & PECT \\
\hline IND & 6.0 & 0.0 & 0.0 & 0.5 & 0.0 & 0.0 & 0.0 & 20.0 \\
\hline $\mathrm{M}-\mathrm{R}$ & 13.3 & 99.1 & 100.0 & 0.3 & 0.0 & 54.0 & 75.0 & 45.7 \\
\hline V-P & 91.1 & 0.0 & 0.0 & 99.5 & 100.0 & 65.0 & 30.9 & 22.9 \\
\hline CIT & 97.7 & 31.9 & 0.0 & 99.5 & 93.7 & 0.0 & 91.2 & 45.7 \\
\hline $\mathrm{H}_{2} \mathrm{~S}$ & 0.0 & 0.0 & 0.0 & 0.0 & $\cdot 0.0$ & 0.0 & 0.0 & 0.0 \\
\hline URE & 94.5 & 9.5 & 0.0 & 64.7 & 2.7 & 3.0 & 4.4 & 21.4 \\
\hline $\mathrm{KCN}$ & 97.9 & 88.0 & 100.0 & 98.0 & 98.7 & 97.0 & 98.5 & 56.1 \\
\hline MOT & 0.0 & 0.0 & 0.0 & 94.5 & 97.3 & 93.0 & 97.1 & 55.7 \\
\hline GEL & 3.3 & 0.0 & 0.0 & 1.0 & 0.0 & 0.0 & 98.5 & 67.1 \\
\hline LYS & 97.2 & 48.0 & 0.0 & 0.5 & 98.7 & 100.0 & 82.0 & 0.0 \\
\hline ARG & 0.9 & 6.0 & 0.0 & 96.5 & 0.0 & 9.0 & 4.4 & 5.7 \\
\hline ORN & 0.0 & 4.0 & 0.0 & 96.0 & 98.7 & 100.0 & 98.5 & 0.0 \\
\hline P-D & 0.0 & 0.0 & 0.0 & 0.0 & 0.0 & 0.0 & 1.5 & 0.0 \\
\hline MAL & 92.5 & 4.0 & 95.5 & 80.6 & 74.7 & 74.0 & 7.4 & 21.4 \\
\hline GAS & 96.5 & 64.0 & 0.0 & 100.0 & 100.0 & 100.0 & 94.1 & 17.1 \\
\hline LAC & 98.2 & 24.1 & 0.0 & 93.5 & 92.1 & 0.0 & 1.5 & 52.9 \\
\hline SUC & 98.9 & 16.3 & 68.2 & 96.5 & 100.0 & 12.0 & 100.0 & 74.3 \\
\hline MAN & 100.0 & 100.0 & 100.0 & 100.0 & 100.0 & 100.0 & 100.0 & 75.7 \\
\hline DUL & 31.5 & 0.0 & 0.0 & 12.9 & 4.0 & 1.0 & 0.0 & 0.0 \\
\hline SAL & 99.7 & 97.4 & 100.0 & 75.6 & 98.7 & 13.0 & 100.0 & 70.0 \\
\hline $\mathrm{ADO}$ & 87.7 & 98.3 & 100.0 & 28.4 & 98.7 & 0.0 & 8.8 & 0.0 \\
\hline INO & 91.9 & 58.6 & 95.5 & 21.9 & 100.0 & 0.0 & 97.0 & 4.3 \\
\hline SOR & 99.4 & 78.0 & 100.0 & 94.5 & 100.0 & 0.0 & 97.0 & 2.8 \\
\hline ARA & 99.9 & 100.0 & 100.0 & 99.5 & 100.0 & 96.0 & 92.6 & 70.0 \\
\hline RAF & 99.7 & 90.0 & 68.2 & 97.0 & 96.0 & 0.0 & 86.8 & 65.7 \\
\hline RHM & 99.3 & 60.0 & 95.5 & 92.0 & 98.7 & 93.0 & 0.0 & 64.3 \\
\hline ONP & $99.6^{e}$ & $94.8^{e}$ & $100.0^{e}$ & $99.0^{e}$ & $97.4^{e}$ & $100.0^{e}$ & $100.0^{e}$ & $71.4^{e}$ \\
\hline $\mathrm{ESC}$ & 98.9 & 75.0 & 15.4 & 29.3 & 98.0 & 6.0 & 73.3 & 90.0 \\
\hline
\end{tabular}


TABLE $1-$ Continued

\begin{tabular}{|c|c|c|c|c|c|c|c|}
\hline \multirow{2}{*}{$\begin{array}{l}\text { Biochemical } \\
\text { tests }\end{array}$} & \multicolumn{7}{|c|}{ Bacterial taxa } \\
\hline & SERR & PVUL & PMIR & PMOR & PRET & PRVA & PRVB \\
\hline IND & 0.2 & 98.2 & 1.9 & 100.0 & 100.0 & 99.5 & 98.7 \\
\hline M-R & 17.7 & 93.0 & 98.8 & 97.1 & 93.3 & 99.8 & 100.0 \\
\hline V-P & 100.0 & 0.0 & 15.6 & 0.0 & 0.0 & 0.0 & 0.0 \\
\hline CIT & 98.6 & 10.5 & 58.7 & 0.0 & 95.6 & 97.9 & 95.6 \\
\hline $\mathrm{H}_{2} \mathrm{~S}$ & 0.0 & 94.7 & 94.2 & 0.0 & 0.0 & 0.0 & 0.0 \\
\hline URE & 29.1 & 94.7 & 88.4 & 98.2 & 100.0 & 0.0 & 0.0 \\
\hline $\mathrm{KCN}$ & 99.1 & 100.0 & 98.6 & 99.0 & 96.7 & 98.8 & 98.6 \\
\hline MOT & 98.6 & 94.7 & 95.9 & 87.7 & 94.4 & 96.5 & 87.0 \\
\hline GEL & 86.9 & 90.6 & 91.8 & 0.0 & 0.0 & 0.0 & 0.0 \\
\hline LYS & 99.6 & 0.0 & 0.0 & 0.0 & 0.0 & 0.0 & 0.0 \\
\hline ARG & 1.3 & 0.0 & 0.0 & 0.0 & 0.0 & 0.0 & 0.0 \\
\hline ORN & 99.4 & 0.0 & 99.2 & 97.1 & 0.0 & 1.4 & 0.0 \\
\hline P.D & 2.7 & 100.0 & 99.6 & 95.3 & 97.8 & 97.2 & 93.7 \\
\hline MAL & 1.7 & 0.0 & 1.5 & 4.8 & 1.2 & 0.7 & 1.2 \\
\hline GAS & 52.6 & 86.0 & 93.4 & 84.9 & 12.2 & 85.8 & 0.0 \\
\hline LAC & 2.2 & 0.0 & 1.5 & 0.0 & 8.9 & 0.3 & 3.8 \\
\hline SUC & 99.7 & 94.7 & 18.9 & 1.0 & 13.3 & 13.0 & 2.6 \\
\hline MAN & 100.0 & 0.0 & 0.0 & 0.0 & 88.5 & 2.0 & 13.3 \\
\hline DUL & 0.0 & 0.0 & 0.0 & 0.0 & 0.0 & 0.0 & 0.0 \\
\hline SAL & 95.1 & 58.2 & 0.8 & 0.0 & 30.0 & 0.6 & 1.9 \\
\hline ADO & 55.5 & 0.0 & 0.0 & 0.0 & 80.9 & 94.5 & 3.8 \\
\hline INO & 78.5 & 0.0 & 0.0 & 0.0 & 93.3 & 0.6 & 97.5 \\
\hline SOR & 98.3 & 0.0 & 0.0 & 0.0 & 1.2 & 0.0 & 4.1 \\
\hline ARA & 0.0 & 0.0 & 0.0 & 0.0 & 0.0 & 0.7 & 5.0 \\
\hline RAF & 1.7 & 0.0 & 1.0 & 0.0 & 9.5 & 1.0 & 6.2 \\
\hline RHM & 0.0 & 9.4 & 1.5 & 0.0 & 67.9 & 0.0 & 0.0 \\
\hline ONP & 100.0 & 0.0 & 1.9 & $0.0^{e}$ & $10.0^{e}$ & $0.3^{e}$ & $3.8^{e}$ \\
\hline ESC & 90.8 & 59.0 & 0.0 & 0.0 & 30.4 & 0.0 & 0.0 \\
\hline
\end{tabular}

a The bacterial taxa are abbreviated as follows: Escherichia coli, ESCH; Shigella sp., SHIG; Edwardsiella tarda, EDWR; Salmonella cholerae-suis, CSUI; Salmonella typhi, TYPH; Salmonella enteritidis, ENTR; Arizona hinshawii, ARIZ; Citrobacter freundii, CITR; Klebsiella pneumoniae, KPNU; Klebsiella ozaenae, KOZE; Klebsiella rhinoschleromatis, KRHI; Enterobacter cloacae, ECLO; Enterobacter aerogenes, EAER; Enterobacter hafniae, EHAF; Serratia liquefaciens, ELIQ; Pectobacterium caratovorum, PECT; Serratia marcescens, SERR; Proteus vulgaris, PVUL; Proteus mirabilis, PMIR; Proteus morganii, PMOR; Proteus rettgeri, PRET; Providencia alcalifaciens, PRVA; Providencia stuartii, PRVB.

${ }^{\circ}$ The biochemical tests are abbreviated as follows: indole, IND; methyl red, M-R; Voges-Proskauer, V-P; citrate (Simmons'), CIT; $\mathrm{H}_{2} \mathrm{~S}$ (TSI), $\mathrm{H}_{2} \mathrm{~S}$; urea, URE; KCN, KCN; motility, MOT; gelatin, GEL; lysine decarboxylase, LYS; arginine dihydrolase, ARG; ornithine decarboxylase, ORN; phenylalanine deaminase, P-D; malonate, MAL; gas from glucose, GAS; acid from lactose, LAC; acid from sucrose, SUC. acid from mannitol, MAN; acid from dulcitol, DUL; acid from salicin, SAL; acid from adonitol, ADO; acid from inositol, INO; acid from sorbitol, SOR; acid from arabinose, ARA; acid from raffinose, RAF; acid from rhamnose, RHM; ONPG hydrolysis, ONP; esculin hydrolysis, ESC.

${ }^{c}$ Incubation is 48 hours at 35 to $37 \mathrm{C}$ except for gelatin at $25 \mathrm{C}$. Data compiled from references 3-7, 11-13.

${ }^{d}$ The three-species concept of Salmonella recommended by Ewing (2) was employed in this study.

e In certain cases, percent positive data were not available for ONPG. These data represent lactose percent positive, including all delayed reactions.

tests on one taxonomic unit to produce one hypothetical culture. The process was repeated to produce 100 hypothetical cultures of each taxonomic unit.

Two evaluations were then performed on the hypothetical cultures. The first determined how much inaccuracy was introduced in the generation process, and the second tabulated the incidence of atypical reactions in each "taxon" of hypothetical cultures. These results were then compared with computed predictions to determine correspondence between observed and predicted behavior of the generating program.
The first evaluating program summed the number of positive results for each test in each taxonomic unit of the hypothetical cultures. That is, it determined the percent positive of each test in each taxonomic unit in the set of hypothetical cultures. These percent positive values were subtracted from the corresponding numbers in the data base to determine how closely the simulation resembled the data base. For example, the data base percent positive values (Table 1) for eight representative tests (ADO through ESC) performed on Escherichia are 5.6, 1.1, 93.4, 99.4, 50.9, $82.3,95.9$, and 30.0 . The corresponding values found 
in the hypothetical cultures are $3.0,1.0,92.0,100.0$, $52.0,82.0,95.0$, and 34.0. Subtracting the second set of values from the first gives the differences 2.6, 0.1, $1.4,-0.6,-1.1,0.3,0.9$, and -3.1 . The absolute sum of these differences (considering all values as positive) is 10.1, and the net sum (of positive and negative values) is 0.5 . The absolute sum of differences over all 28 tests for this taxon is 44.9 , and the corresponding net value is -10.7 (see Table 2).

The predicted mean (long run average) values of these absolute differences were computed by summing the binomial probabilities of their components:

$$
D=\sum_{x=0}^{100}\left(P_{x}|p-x|\right)
$$

where $D$ is the predicted average absolute difference between a data base percent positive value $p$ and its corresponding value in the set of hypothetical cultures for a particular (test:taxon) combination; $x$ is a variable with range 0 to 100 ; and $P_{x}$ is the binomial probability (expected long-term frequency) of an individual value $x$ in a binomial distribution of size $N$ $=100$ and percentage $p$.

Thus, for each trial value of $p$ (percent positive), each possible absolute deviation $(|p-x|)$ was multiplied by its binomial probability $\left(P_{x}\right)$ and the products were summed. A program was written which computed values of $D$ for all integer values of $p$ from 1 through 50 . $D$ values from 1 to 50 were sufficient, as those from 51 to 100 are a "mirror image."

Data base values for percent positive or 100 - percent positive, whichever was smaller, were rounded to integers, and the corresponding values of $D$ were summed for all tests belonging to each taxon to obtain the predicted absolute difference between the data base and the simulation for each taxon. For example, Salmonella typhi (TYPH) is variable in two tests, $\mathrm{H}_{2} \mathrm{~S}$ and ARG, with rounded (100-percent positive) values of 6 and 19 and corresponding D values of 1.868661 and 3.115799 . These values were summed and rounded to one decimal place, giving a result of 5.0 (Table 2 ).

The second evaluation (tabulation) program compared each hypothetical culture with a master card containing "typical reactions" for the corresponding taxonomic unit, and counted the discrepancies (atypical reactions). The range and mean of these observed atypical reactions were determined for each taxonomic unit. The predicted mean number of atypical reactions was computed for each taxon by summing together the smaller of percent positive or 100-percent

TABLE 2. Analysis of discrepancies between percent positive of hypothetical bacteria and data base ${ }^{a}$

\begin{tabular}{|c|c|c|c|c|c|c|c|c|c|}
\hline \multirow[t]{2}{*}{ No. } & \multirow[t]{2}{*}{ Taxon $^{b}$} & \multicolumn{2}{|c|}{$\begin{array}{l}\text { Sum of absolute } \\
\text { differences }\end{array}$} & \multirow{2}{*}{$\begin{array}{l}\text { Predicted } \\
\text { minus } \\
\text { observed }\end{array}$} & \multirow{2}{*}{$\begin{array}{c}\text { No. of } \\
\text { tests }>0.5 \\
\text { and }<95.5\end{array}$} & \multicolumn{2}{|c|}{$\begin{array}{c}\text { Absolute } \\
\text { differences } \\
\text { per variable } \\
\text { test }\end{array}$} & \multirow[t]{2}{*}{$\begin{array}{l}\text { Sum of net } \\
\text { differences }\end{array}$} & \multirow{2}{*}{$\begin{array}{c}\text { Maximum } \\
\text { difference } \\
\text { in a single } \\
\text { test }\end{array}$} \\
\hline & & Predicted & Observed & & & $\begin{array}{c}\text { Pre- } \\
\text { dicted }\end{array}$ & $\left|\begin{array}{c}\text { Ob- } \\
\text { served }\end{array}\right|$ & & \\
\hline 1 & ESCH & 50.1 & 44.9 & 5.2 & 20 & 2.5 & 2.2 & -10.7 & -10.8 \\
\hline 2 & SHIG & 32.4 & 23.0 & 9.4 & 12 & 2.7 & 1.9 & -13.8 & -3.5 \\
\hline 3 & EDWR & 4.8 & 6.9 & -2.1 & 4 & 1.2 & 1.7 & -1.7 & -3.7 \\
\hline 4 & CSUI & 14.1 & 28.0 & -13.9 & 5 & 2.8 & 5.6 & -12.0 & -14.0 \\
\hline 5 & TYPH & 5.0 & 3.0 & 2.0 & 2 & 2.5 & 1.5 & -2.4 & -2.7 \\
\hline 6 & ENTR & 24.5 & 24.8 & -0.3 & 15 & 1.6 & 1.7 & -9.4 & -9.2 \\
\hline 7 & ARIZ & 25.2 & 25.0 & 0.2 & 15 & 1.7 & 1.7 & -9.6 & -4.0 \\
\hline 8 & CITR & 49.7 & 35.3 & 14.4 & 21 & 2.4 & 1.7 & 10.3 & 6.4 \\
\hline 9 & KPNU & 30.3 & 29.0 & 1.3 & 19 & 1.6 & 1.5 & 10.6 & 7.7 \\
\hline 10 & KOZE & 49.4 & 60.0 & -10.6 & 19 & 2.6 & 3.2 & 29.0 & 7.9 \\
\hline 11 & KRHI & 15.4 & 15.1 & 0.3 & 6 & 2.6 & 2.5 & -10.7 & -9.8 \\
\hline 12 & ECLO & 43.4 & 48.9 & -5.5 & 23 & 1.9 & 2.1 & 2.7 & 8.9 \\
\hline 13 & EAER & 20.0 & 19.1 & 0.9 & 15 & 1.3 & 1.3 & 6.1 & 3.0 \\
\hline 14 & EHAF & 29.6 & 33.0 & -3.4 & 13 & 2.3 & 2.5 & -11.0 & -10.0 \\
\hline 15 & ELIQ & 39.2 & 38.7 & 0.5 & 20 & 2.0 & 1.9 & -11.5 & -6.0 \\
\hline 16 & PECT & 72.0 & 58.2 & 13.8 & 22 & 3.3 & 2.6 & -24.8 & -11.3 \\
\hline 17 & SERR & 33.8 & 27.5 & 6.3 & 18 & 1.9 & 1.5 & 10.3 & 5.9 \\
\hline 18 & PVUL & 27.5 & 27.7 & -0.2 & 12 & 2.3 & 2.3 & -10.3 & -8.0 \\
\hline 19 & PMIR & 29.1 & 40.6 & -11.5 & 18 & 1.6 & 2.3 & 2.2 & -10.1 \\
\hline 20 & PMOR & 14.0 & 12.9 & 1.1 & 9 & 1.6 & 1.4 & -8.9 & -3.1 \\
\hline 21 & PRET & 40.2 & 44.9 & -4.7 & 18 & 2.2 & 2.5 & -7.9 & -7.5 \\
\hline 22 & PRVA & 18.1 & 25.8 & -7.7 & 15 & 1.2 & 1.7 & -0.4 & -5.2 \\
\hline 23 & PRVB & 24.4 & 23.8 & 0.6 & 16 & 1.5 & 1.5 & -6.2 & 6.0 \\
\hline Sum & & 692.2 & 696.1 & -3.9 & 337 & & & -80.1 & \\
\hline Mean & & 30.1 & 30.3 & & 14.7 & 2.1 & 2.1 & & \\
\hline S.D. & & 16.0 & 14.8 & & 6.0 & 0.6 & 0.9 & & \\
\hline
\end{tabular}

${ }^{a}$ Discrepancies among all tests summed for each taxonomic unit.

${ }^{b}$ For abbreviations, see Table 1 , footnote $a$. 
positive for each of the 28 tests and dividing by 100 . For Escherichia, the first 8 numbers chosen in this way are $1.4,0.1,0.0,0.2,0.0,0.0,2.4$, and 30.9 . Their sum is 35.0 , and the predicted mean frequency of atypical reactions in tests IND through MOT is 0.35 per culture.

The predicted range of atypical reactions was estimated by assuming that they were normally distributed. Using this assumption, the variance for a taxon was computed as the sum of (percent positive/100) $\times(100$-percent positive/100) for each test used.

The predicted range was obtained as predicted mean 2.576 standard deviations (99\% limits), rounded to integer values (Table 3 ).

All programs were written in Fortran IV and run on IBM $360-30$ or $360-67$ computers, except for the determination of average deviations in a binomial of size 100 , which was written in Fortran $\mathrm{V}$ and run on a Univac 1108 computer. Conventional calculations were performed on mechanical and electronic calculators.

\section{RESULTS}

One hundred hypothetical cultures were constructed for each of 23 taxonomic units (taxa). Each culture is defined by 28 simulated biochemical test results $(100 \times 23 \times 28=64,400$ total test results simulated). The output is in the form of punched IBM cards.

Table 2 summarizes the observed and predicted discrepancies between the data base and the 2,300 hypothetical cultures. In this table, the observed absolute differences computed by the first evaluating program and the predicted absolute differences (computed by summing their binomial probabilities) are separately summed for all tests in each taxon. The total of observed absolute differences for all taxa is 696.1 , for an error rate of $696.1 / 64,400=0.0108$ discrepant reactions per individual test (i.e., about $1.1 \%$ ). The predicted total is 692.2 $(1.07 \%)$. The number of tests classed as variable (data base values $>0.5$ and $<99.5$ ) range from 2 (S. typhi) to 23 (Enterobacter cloacae) and total 337 for all taxa, out of a possible $28 \times 23=644$ if all tests were variable in all taxa.

The observed differences between data base and simulated values are also summed as net values, and the largest difference found between the data base and 100 simulations of a single test is shown for each taxon. The sum of net

TABLE 3. Frequency analysis of atypical reactions among 100 hypothetical cultures of each taxonomic unit

\begin{tabular}{|c|c|c|c|c|c|c|c|c|}
\hline \multicolumn{8}{|c|}{ Atypical reactions per hypothetical culture } & \multirow{3}{*}{$\begin{array}{c}\begin{array}{c}\text { Test of } \\
\text { differences } \\
\text { between means }\end{array} \\
Z=\sqrt{\frac{X}{X_{p}}-\bar{X} o} \\
S_{p}{ }^{2}+S_{o}^{2}\end{array}$} \\
\hline \multirow{2}{*}{ No. } & \multirow{2}{*}{ Taxon $^{a}$} & \multicolumn{2}{|c|}{ Mean } & \multicolumn{2}{|c|}{ Variance } & \multicolumn{2}{|c|}{ Range } & \\
\hline & & Predicted & Observed & Predicted & Observed & Predicted $^{b}$ & Observed & \\
\hline 1 & ESCH & 3.75 & 3.46 & 2.45 & 2.76 & $0-8$ & $0-7$ & 0.13 \\
\hline 2 & SHIG & 2.10 & 2.26 & 1.57 & 1.31 & $0-5$ & $0-5$ & -0.09 \\
\hline 3 & EDWR & 0.13 & 0.18 & 0.13 & 0.17 & $0-1^{c}$ & $0-2$ & -0.09 \\
\hline 4 & CSUI & 1.05 & 0.97 & 0.70 & 0.66 & $0-3$ & $0-3$ & 0.07 \\
\hline 5 & TYPH & 0.24 & 0.22 & 0.21 & 0.17 & $0-1^{c}$ & $0-1$ & 0.03 \\
\hline 6 & ENTR & 0.96 & 1.06 & 0.68 & 0.54 & $0-3$ & $0-3$ & -0.09 \\
\hline 7 & ARIZ & 1.03 & 0.86 & 0.84 & 0.65 & $0-3$ & $0-3$ & 0.14 \\
\hline 8 & CITR & 3.12 & 3.05 & 2.27 & 2.17 & $0-7$ & $0-6$ & 0.03 \\
\hline 9 & KPNU & 1.14 & 1.18 & 0.97 & 0.90 & $0-4$ & $0-5$ & -0.03 \\
\hline 10 & KOZE & 3.41 & 3.14 & 1.54 & 2.81 & $0-7$ & $0-8$ & 0.13 \\
\hline 11 & KRHI & 0.93 & 0.83 & 0.69 & 0.75 & $0-3$ & $0-3$ & 0.08 \\
\hline 12 & ECLO & 2.18 & 2.07 & 1.70 & 1.26 & $0-6$ & $0-5$ & 0.06 \\
\hline 13 & EAER & 0.65 & 0.68 & 0.64 & 0.52 & $0-3^{c}$ & $0-3$ & -0.03 \\
\hline 14 & EHAF & 1.72 & 1.87 & 1.26 & 1.29 & $0-5$ & $0-5$ & -0.09 \\
\hline 15 & ELIQ & 1.77 & 1.78 & 1.46 & 1.53 & $0-5$ & $0-5$ & -0.01 \\
\hline 16 & PECT & 5.94 & 5.83 & 3.94 & 3.98 & $1-11$ & $2-12$ & 0.05 \\
\hline 17 & SERR & 2.04 & 2.09 & 1.42 & 1.84 & $0-5$ & $0-7$ & -0.03 \\
\hline 18 & PVUL & 1.56 & 1.50 & 1.15 & 0.98 & $0-4$ & $0-4$ & 0.04 \\
\hline 19 & PMIR & 1.26 & 1.47 & 1.00 & 0.98 & $0-4$ & $0-4$ & -0.15 \\
\hline 20 & PMOR & 0.47 & 0.34 & 0.42 & 0.25 & $0-2$ & $0-2$ & 0.16 \\
\hline 21 & PRET & 2.08 & 2.33 & 1.67 & 1.80 & $0-5$ & $0-5$ & -0.13 \\
\hline 22 & PRVA & 0.51 & 0.45 & 0.46 & 0.35 & $0-2$ & $0-3$ & 0.07 \\
\hline 23 & PRVB & 0.75 & 0.83 & 0.69 & 0.65 & $0-3$ & $0-4$ & -0.07 \\
\hline
\end{tabular}

${ }^{a}$ For abbreviations, see Table 1 , footnote $a$.

${ }^{\circ}$ Rounded to nearest integer.

c The same results were obtained from a cumulative Poisson distribution table. 
differences over all taxa is -80.1 . This yields a mean net difference of $-80.1 / 2,300=-0.0348$ per culture, and $-0.0348 / 28=-0.0012$ per individual reaction (i.e., about $0.1 \%$ ). The negative sign indicates that positive reactions are observed $0.1 \%$ less frequently in the data base than in the simulation.

Table 3 compares the mean frequency, variance, and range of atypical reactions (those expected to occur less than $50 \%$ of the time) found in the 2,300 hypothetical cultures, with their predicted values. Assuming the (predicted mean - observed mean) differences to be normally distributed, $\mathrm{Z}$ was computed as:

$$
\left.\left(\bar{X}_{p}-\bar{X}_{o}\right) / \sqrt{S_{p}{ }^{2}+S_{o}{ }^{2}}\right) .
$$

All $\mathrm{Z}$ values are well within the $\pm 1.645(5 \%$ significance) range; thus, the predicted and observed means are in good agreement.

\section{DISCUSSION}

The data in Table 1 are for $48 \mathrm{~h}$ of incubation. This was selected as probably more typical of average diagnostic laboratory procedure than a longer period.

The data in Table 2 show the degree to which the hypothetical cultures of each taxonomic unit resemble the data base (Table 1) from which they were generated, and compare this with the behavior predicted by binomial probabilities. For example, the observed absolute total of discrepancies between the data base and the 100 hypothetical cultures of Escherichia was 44.9. This indicates that the simulation differed from the data base by the equivalent of 45 individual biochemical tests out of a total of 2,800 (100 hypothetical cultures $\times 28$ individual tests), or $45 / 2,800=1.6 \%$ total error per individual test. The predicted frequency of absolute differences between the data base and the hypothetical cultures was 50.1 for this taxon, or $50.1 / 2,800=1.8 \%$ error per test. Twenty of the 28 tests were $0.5 \%$ or more variable within the taxon, giving a mean predicted difference of $50.1 / 2,000=2.5 \%$ per variable test.

Agreement of predicted and observed absolute differences ranged from a (predicted observed) error of 0.2 for Arizona to 14.4 for Citrobacter. Summation of the differences for all taxa, representing a total of 337 variable test $\boldsymbol{x}$ taxon combinations, yielded an error of only -3.9 between the predicted and observed totals. Agreement of mean values was also close (Table 2).

It is readily seen that the prediction of absolute discrepancies is controlled by two factors: the number of variable reactions in a taxon (defined as percent positive values $>0.5$ and $<95.5$ ), and the degree to which they tend to approach 50.0 , the value at which the binomial distribution predicts the greatest number of errors.

To indicate the effect of different numbers of variable tests in different taxa, the absolute differences per variable test are also shown. These differences are more uniform from taxon to taxon. The remaining variability is due to differing percent positive values among the variable tests.

The net differences are calculated in order to examine the positive or negative error in each taxonomic unit as a whole. In each case, these totals were less than the absolute totals, i.e., no taxonomic unit of 100 hypothetical cultures contained exclusively positive or negative discrepancies from the data base. The maximum discrepancy for a single test in each taxon is shown to indicate the degree to which discrepancies in one test (on 100 hypothetical cultures) contributed to the sum of absolute discrepancies in each taxon. For example, the maximum observed difference between the data base and the hypothetical percent positive values for Escherichia was -10.8. This was found in the ornithine test. Predicted single-test absolute differences $(D)$ depend entirely on the value of $p$ (the lesser of percent positive or 100-percent positive) and range from 0.732 for $p=1$ to 3.98 for $p=50$. The mean observed absolute difference is 30.3 errors per taxon ( $1.1 \%$ of 2,800 tests) and the largest observed absolute difference is $60.0(2.1 \%$ of 2,800 tests, and $3.2 \%$ of 1,900 variable tests) for Klebsiella ozaenae. The largest difference predicted is 72.0 for Pectobacterium $(2.6 \%$ of 2,800 tests and $3.3 \%$ of 2,200 variable tests). The hypothetical culture simulation is considered satisfactory, since it is generally in agreement with predicted behavior, and the errors are smaller than those inherent in many of the biochemical tests, due to species and procedural variability, as seen in Table 1. The agreement between predicted and observed frequencies of atypical reactions (Table 3 ) is similarly satisfactory.

As an example of operations which can be performed with the generating program, it is used to simulate the average number of atypical reactions (reactions occurring less than $50 \%$ of the time) which can be expected if all 28 biochemical tests are to be run on a large number of cultures belonging to taxa represented in the data base. Table 3 summarizes the results of this experiment. It is evident that certain taxa are more variable than others, whether this variability is measured by the 
range of the number of atypical reactions (reactions expected to occur less than $50 \%$ of the time), the mean number of atypical reactions, or the percent of hypothetical cultures with one or more atypical reactions. Comparison with Table 1 illustrates this point. Thus, the hypothetical Pectobacterium, Escherichia, K. ozaenae, and Citrobacter freundii have mean atypical reactions (Table 3 ) of $5.83,3.46,3.14$, and 3.05 per culture, respectively, and the number of tests in Table 1 with percent positive values $<90$ and $>10$ for these taxa are $18,9,10$, and 10 , respectively (relatively variable). On the other hand, Edwardsiella (0.18 mean atypical reactions) and $S$. typhi $(0.22)$ are relatively invariant. They have 0 and 1 tests, respectively, with data base values $<90$ and $>10$.

Results of this approach can have practical value by indicating the expected variability of cultures identified in routine laboratory practice. For example, Table 3 indicates that $K$. pneumoniae has 1.18 observed atypical reactions per culture (1.14 predicted). This is the variability to be expected in this taxon with these tests. To be valid, such a simulation should be performed with the same tests which are actually employed in the user's laboratory. The computed prediction can also be used for this purpose and is less cumbersome if only mean values and ranges are required. Individual hypothetical cultures are needed, however, for certain experimental designs.

The hypothetical cultures are presently being used to define the performance of an experimental program for computer-assisted identification of Enterobacteriaceae. The taxonomy which was current at the time the programs were developed has been retained in this study. Recent publications have proposed the taxa Citrobacter diversus, Enterobacter agglomerans, and Serratia rubidaea; new data on $E$. coli are also available (7-10). Recomputation with an updated data base should lead to an improved representation of the Enterobacteriaceae. Printouts of the programs and hypothetical cultures described in this report are available on request from the author.

\section{ACKNOWLEDGMENTS}

I am grateful to I. Huber, Chairman of my faculty committee; T. Hayton and F. H. Midlige, committee members, for their advice in this work; to R. Lamm, Warner
Lambert Research Institute, for computation of average binomial deviations; to the Fairleigh Dickinson Computer Center (Teaneck) and the Department of Mathematics (Madison) for computer time; and to Technical Information Services, Warner Lambert Research Institute, for keypunch and card reproducing facilities.

\section{REPRINT REQUESTS}

Address reprint requests to: W. D. Young, Jr., 68 Gates Avenue, Montclair, N. J. 07042.

\section{LITERATURE CITED}

1. Camin, J. H., and R. R. Sokal. 1965. A method for deducing branching sequences in phylogeny. Evolution 19:311-326.

2. Ewing, W. H. 1963. An outline of nomenclature for the family Enterobacteriaceae. Int. Bull. Bacteriol. Nomen. Taxon. 13:95-110.

3. Ewing, W. H. 1965. Differentiation of members of the genera Salmonella, Arizona, and Citrobacter by biochemical methods. National Center for Disease Control, Atlanta.

4. Ewing, W. H. 1966. Enterobacteriaceae: taxonomy and nomenclature. National Center for Disease Control, Atlanta.

5. Ewing, W. H. 1969. Biochemical reactions given by Enterobacteriaceae in commonly used tests. National Center for Disease Control, Atlanta.

6. Ewing, W. H. 1970. Differentiation of Enterobacteriaceae by biochemical reactions. Revised and emended. National Center for Disease Control, Atlanta.

7. Ewing, W. H. 1971. Biochemical characterization: Citrobacter freundii and Citrobacter diversus. National Center for Disease Control, Atlanta.

8. Ewing, W. H., B. R. Davis, and W. J. Martin. 1972. Biochemical characterization: Escherichia coli. National Center for Disease Control, Atlanta.

9. Ewing, W. H., B. R. Davis, and M. A. Fife. 1972. Biochemical characterization of Serratia liquefaciens and Serratia rubidaea. National Center for Disease Control, Atlanta.

10. Ewing, W. H., and M. A. Fife. 1971. Enterobacter agglomerans. National Center for Disease Control, Atlanta.

11. Fife, M. A., W. H. Ewing, and B. R. Davis. 1965. The biochemical reactions of the tribe Klebsiellae. National Center for Disease Control, Atlanta.

12. Lubin, A. H., and W. H. Ewing. 1964. Studies on the beta-d-galactosidase activities of Enterobacteriaceae. Public Health Lab. 22:83-101.

13. Marymont, J. H., Jr., U. Amanna, and B. H. Lloyd. 1966. Usefulness of $\beta$-galactosidase demonstration in diag. nostic bacteriology. Tech. Bull. Reg. Med. Technol. 36:292-293.

14. Moss, W. W. 1971. Taxonomic repeatability: an experimental approach. Syst. Zool. 20:309-330.

15. Rohlf, F. J., and R. R. Sokal. 1967. Taxonomic structure from randomly and systematically scanned biological images. Syst. Zool. 16:246-260.

16. Sneath, P. H. A., and R. R. Sokal. 1973. Numerical taxonomy. W. H. Freeman and Co., San Francisco.

17. Sokal, R. R., and P. H. A. Sneath. 1963. Principles of numerical taxonomy. W. H. Freeman and Co., San Francisco. 